\title{
MATHEMATISCHE ZEITSCHRIFT
}

UNTER STÄDIGER MITTIRKUNG VON
E. KAM KE
TUUBINGEN
K. KN OPP
TÜBINGEN
R. NEVANLINNA
HELSINKI
E. SCHMIDT
BERLIN
F. K. SCHM IDT
HEIDELBERG

HERAUSGEGEBEN VON

\section{H. WIELANDT}

TUUBINGEN

WISSENSCHAFTLICHER BEIRAT

W. BLASCHKE L. FEJER A.E. INGHAM

H.KNESER W.MAGNUS O.PERRON W.SÜSS

64. BAND

BERLIN • GOTTINGEN • HEIDELBERG

S P R I N G E R - V E R L A G

1956 


\section{Inhalt des 64. Bandes}

BAUMAN, V., Eine nichtlineare Integrodifferentialgleichung der Wärmeübertragung bei Wärmeleitung und -strahlung. . . . . . . . . . . . . . . . . . . . . 353

BeHRExs, E.-A., Zur additiven Idealtheorie in nichtassoziativen Ringen . . . . . . 169

¿CARLiTz, L., Arithmetic properties of elliptic functions . . . . . . . . . . . . . 425

EвEL, I., Analytische Bestimmung der Darstellungsanzahlen natürlicher Zahlen durch spezielle ternäre quadratische Formen mit Kongruenzbedingungen . . . 217

Eichler, M., Der Hilbertsche Klassenkörper eines imaginärquadratischen Zahlliörpers . . . . . . . . . . . . . . . . . . . . . . . . . . . . . 229

GaIER, D., Über die Äquivalenz der $B_{k} \mid$-Verfahren . . . . . . . . . . . . . . . 183

Gaier, D., Uber die konforme Abbildung veränderlicher Gebiete . . . . . . . . . 385

Groor, J. DE, A System of Continuous, Mutually Non-differentiable Functions . . 192

Gundiach, K.-B., Poincarésche und Eisensteinsche Reihen zur Hilbertschen Modulgruppe . . . . . . . . . . . . . . . . . . . . . . . . . . . . 339

Herrmaxi, O., Uber den Rang der Schar der Spitzenformen zu Hilbertschen Modulgruppen. . . . . . . . . . . . . . . . . . . . . . . . . . . . . . . . 457

Hsiuxg, $\mathrm{CH}_{\text {- }} \mathrm{CH}_{\mathrm{H}}, \mathrm{A}$ theorem on surfaces with a closed boundary . . . . . . . . . 41

Huppert, B., Über die Auflösbarkeit faktorisierbarer Gruppen. III . . . . . . . . 138

JAcoBs, K., Ergodentheorie und fastperiodische Funktionen auf Halbgruppen . . . 298

Jurkat, W., und A. Peyerimhoff, Lokalisation bei absoluter Cesàro-Summierbarkeit von Potenzreihen und trigonometrischen Reihen. II. . . . . . . . . . . 151

Karzel, H., Über eine Anordnungsbeziehung am Dreieck . . . . . . . . . . . . 131

KAsch, F., Abschätzung der Dichte von Summenmengen (Zweite Mitteilung) . . . 243

KRULL, W., Uber geordnete Gruppen von reellen Funktionen . . . . . . . . . . 10

KuNLE, H., Uber T-Figuren in einem quadratischen Komplex . . . . . . . . . . 270

I.AMPRECHT, E., Zetafunktionen symmetrisch-erzeugbarer algebraischer Funktionenkörper mehrerer Veränderlicher . . . . . . . . . . . . . . . . . . . . . 47

LAUGwitz, D., Über einen Abbildungssatz von B. Sz.-Nagy . . . . . . . . . . . 72

McMsn, $T$. J., Oin figures of equilibrium of rotating liquids . . . . . . . . . . . 286

Neumer, W., Zur Konstruktion von Ordnungszahlen. V. . . . . . . . . . . . . 435

Perron, O., Uber Potenzsummen . . . . . . . . . . . . . . . . . . . . . . 103

Purxam, C. R., A Note on Inverses of Differential Operators . . . . . . . . . . . 149 
Ribenborm, P., Sur une note ic Nagata relative à un problème de Krull . . . . 1559

Rrngel, G., Teilungen der Kbene durch Geraden oder topologische Geraden . . .

Singh, V., Convergence Theorems for a Generalized Laplace Integral . . . . . . . 1

Weier, J., Über Zerlegung cindimensionaler Nullstellengebilde . . . . . . . . . . 1115

Wilansky, A., und K. Zeller, Abschnittsbeschränkte Matrixtransformationen;

starke Limitierbarkeit. . . . . . . . . . . . . . . . . . . . . . . . . . 2558

Witt, E., Die Unterringe der freien Lieschen Ringe. . . . . . . . . . . . . . . 11995

Zeller, K., Uber den perfekten Teil von Wirkfeldern . . . . . . . . . . . . . . . . 11223 
$\mathrm{KASCH}, \mathrm{F}$.

Math. Zeitschr. Bd. 64, S. 243-257 (1956)

\title{
Abschätzung der Dichte von Summenmengen
}

\author{
(Zweite Mitteilung) \\ Von
}

\section{FRIEDRICH KASCH}

\section{$\S 1$. Fragestellung}

In einer kürzlich erschienenen Arbeit mit dem gleichen Titel [2] ${ }^{1}$ ) habe ich Abschätzungen der Dichte von Summenmengen, bei denen einer der Summanden eine Basis endlicher Ordnung der natürlichen Zahlen ist, aufgestellt. Richtunggebend für diese Untersuchungen war eine Vermutung von P. ERDös, die folgendes besagt: Ist $\mathfrak{A}$ eine Menge natürlicher Zahlen der Dichte $\alpha$, $\mathfrak{B}$ eine die 0 enthaltende Basis der natürlichen Zahlen der mittleren Ordnung $\lambda$. und $\gamma$ die Dichte der Summenmenge $\mathfrak{C}=\mathfrak{A}+\mathfrak{B}$, dann gilt ${ }^{2}$ )

$$
\gamma \geqq \alpha\left(1+\frac{1-\alpha}{\lambda}\right) \text {. }
$$

In (I) habe ich Verbesserungen der bisherigen Abschätzungen angegeben, ohne jedoch diese Vermutung erreichen zu können.

Inzwischen bemerkte ich, daß man die Schlußweise aus (I) so weit ausbauen kann, daß damit (1.1) für hinreichend kleine $\alpha$ nicht nur erreicht, sondern übertroffen wird. Genauer: $Z u$ jedem $\lambda$ gibt es ein positives Intervall $0<\alpha<\alpha_{\lambda}$, wo eine bessere Abschätzung als (1.1) gilt. Eine entsprechende Aussage besteht auch fïr die asymptotischen Dichten und die asymptotische mittlere Ordnung.

Der Beweis beruht auf einer Verallgemeinerung der in (I) aufgestellten und als Grundformeln bezeichneten Abschätzungen. Aus diesen verallgemeinerten Grundformeln kann dann auf zwei verschiedenen Wegen das genannte Ergebnis hergeleitet werden. Bei dieser Herleitung können wir uns im wesentlichen auf die ausführliche Darstellung in (I) stützen. Es werden hier die gleichen Voraussetzungen und Bezeichnungen wie in (I) benutzt. Außerdem setzen wir hier sogleich $\alpha>0$ und $\lambda>1$ voraus, da sonst diese trivialen Fälle die Formulierung erschweren.

Zum Schluß deuten wir an, wie unsere Überlegungen auf Gitterpunktmengen im $r$-dimensionalen Raum ausgedehnt werden können. Das ist insofern von Interesse, als es bisher nicht gelungen ist, Dichteabschätzungen für die Summenmenge von zwei beliebigen Mengen positiver Dichte in dieser Richtung zu verallgemeinern ${ }^{3}$ ).

1) Diese Arteit wird in folgenden nit (i) zitiert.

2) Siehe [6], S. 7; tatsächlich hat P. ERDös diese Vermutung für die Ordnung $h$ an Stelle von $\lambda$ ausgesprochen.

3) In dieser Richtung liegt in der Literatur lediglich eine Arbeit von L. CHEO [1] vor; dort wird eine Verallgemeinerung der Schnirelmansschen Ungleichung auf den 


\section{§2. Die komplementären Grundformeln}

1. Die Verallgemeinerung gegenüber (I) besteht vor allem darin, daß jetzt an Stelle der Mengen $\mathfrak{A}$ und $\mathfrak{\mathfrak { A }}$ in der Definition der Funktionen $D(m), E(m)$ und $\bar{E}(m)$ beliebige Mengen $\mathfrak{B}, \bar{\complement}$ mit

$$
\mathfrak{A} \leqq \mathfrak{P} \leqq \mathfrak{\Omega} \leqq \mathfrak{C}=\mathfrak{A}+\mathfrak{B}
$$

treten. Dies sei also im folgenden der Fall. Ferner seien $n$ eine feste natürliche Zahl und $m$ eine natürliche Zahl mit $1 \leqq m \leqq n$.

Definitionen:

$D(m)$ sei gleich der Anzahl der verschiedenen Paare $(p, \bar{q})$ mit $p \in \mathfrak{P}(n), \bar{q} \in \overline{\mathfrak{Z}}(n)$ und $\bar{q}-p=m$;

$E(m)$ sei gleich der Anzahl der verschiedenen Paare $(p, q)$ mit $p \in \mathfrak{P}(n), q \in \cong(n)$ und $q-p=m$;

$\bar{E}(m)$ sei gleich der Anzahl der verschiedenen Paare $(\bar{p}, \bar{q})$ mit $\bar{p} \in \overline{\mathfrak{P}}(n), \bar{q} \in \overline{\mathfrak{D}}(n)$ und $\bar{q}-\bar{p}=m$.

Aus diesen Definitionen erhält man unmittelbar die

Folgerungen:

1. $D(m)$ ist gleich der Anzahl der Zahlen $p \in \Re(n)$ mit $p+m \in \bar{\Omega}(n)$;

2. $D(m)$ ist gleich der Anzahl der Zahlen $\bar{q} \in \bar{\Omega}(n)$ mit $\bar{q}-m \in \mathfrak{B}(n)$;

3. $E(m)$ ist gleich der Anzahl der Zahlen $p \in \mathfrak{B}(n)$ mit $p+m \in \mathfrak{Q}(n)$;

4. $\bar{E}(m)$ ist gleich der Anzahl der Zahlen $\bar{q} \in \bar{\Omega}(n)$ mit $\bar{q}-m \in \overline{\mathfrak{P}}(n)$.

Aus der 1. und 3. Folgerung ergibt sich offenbar die Gleichung $D(m)+E(m)$ $=P(n-m)$, also

$$
D(m)=P(n-m)-E(m) .
$$

Entsprechend erhält man aus der 2. und 4. Folgerung $D(m)+\bar{E}(m)=$ $\bar{Q}(n)-\bar{Q}(m)$, also

$$
D(m)=\bar{Q}(n)-\bar{Q}(m)-\bar{E}(m) .
$$

Die weiteren Überlegungen zielen darauf $\mathrm{ab}$, in (2.1) und (2.2) $D(m)$ nach oben und die rechten Seiten nach unten abzuschätzen.

2. Es soll also zunächst $D(m)$ nach oben abgeschätzt werden, wobei die Basiseigenschaft von $\mathfrak{B}$ benutzt wird.

Hilfssatz 1. Ist $t$ eine natürliche Zahl mit $1 \leq t<m$ und $b \in \mathfrak{B}$, dann gilt

$$
\begin{aligned}
D(m) & \leqq D(t)+D(m-t)+Q(n)-P(n), \\
D(b) & \leq C(n)-Q(n)+P(n)-A(n) .
\end{aligned}
$$

zweidimensionalen Fall unter der Voraussetzung vorgenommen, daß eine der beiden betrachteten Mengen alle ganzzahligen Punkte einer der positiven Halbachsen enthält; in diesem Falle läßt sich die lineare Schlußweise anwenden. Darüber hinaus wird gezeigt, daß eine genaue Verallgemeinerung des Satzes von H. B. MANN auf die Ebene nicht möglich ist. 
Beweis. Zum Beweis von (2.3) stützen wir uns auf die 1. Folgerung. Es sei $p$ eine Zahl, die in $D(m)$ gezählt wird, d.h. $p+m=\bar{q} \in \bar{\Omega}(n)$. Ist bereits $p+t \in \bar{\beth}(n)$, dann wird $p$ in $D(t)$ gezählt. Ist jedoch $p+t \in \Omega(n)$, so unterscheiden wir zwei Fälle.

1. Fall. $p+t$ liegt bereits in $\mathfrak{P}(n): p+t=p^{\prime} \in \mathfrak{P}(n)$. Dann wird $p^{\prime}$ in $D(m-t)$ gezählt, denn es ist $p^{\prime}+m-t=p+m=\bar{q} \in \overline{\mathfrak{D}}(n)$. Zu beachten ist dabei, daß die so zugeordneten Zahlen $p^{\prime}$ für verschiedene $p$ verschieden sind.

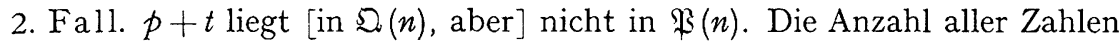
aus $\mathfrak{S}(n)$, die nicht in $\mathfrak{P}(n)$ liegen, ist offenbar gleich $Q(n)-P(n)$. Damit ist (2.3) vollständig bewiesen.

Zum Beweis von (2.4) sei $b \in \mathfrak{B}(n)$ und $p+b=\bar{q} \subseteq \bar{Q}(n)$. Wir unterscheiden wieder zwei Fälle.

1. Fall. $p$ liegt bereits in $\mathfrak{A}(n): p=a$. Dann ist $a+b=\bar{q}$ ein Element aus $\mathfrak{C}(n)$, das nicht in $\mathfrak{(}(n)$ liegt, und die Anzahl aller Elemente aus $\mathfrak{S}(n)$, die nicht in $\mathfrak{\Omega}(n)$ liegen, ist gleich $C(n)-Q(n)$.

2. Fall. $p$ ist ein Element aus $\mathfrak{B}(n)$, das nicht in $\mathfrak{A}(n)$ liegt. Die Anzahl aller derartigen Elemente ist gleich $P(n)-A(n)$. Damit ist (2.4) bewiesen.

Sei nun $m=b_{1}+\cdots+b_{l(m)}\left(b_{i} \in \mathfrak{B}\right)$ eine nach Voraussetzung existierende Darstellung von $m$, so besteht auf Grund von (2.3) und (2.4) die Ungleichung

$$
\begin{aligned}
D(m) & \leqq l(m)\{C(n)-Q(n)+P(n)-A(n)\}+\{l(m)-1\}\{Q(n)-P(n)\} \\
& =l(m)\{C(n)-A(n)\}-\{Q(n)-P(n)\} .
\end{aligned}
$$

Daraus erhält man durch Summation von 1 bis $k \leqq n$ unter Beachtung der Definition der mittleren Ordnung $\lambda$ :

$$
\sum_{m=1}^{k} D(m) \leqq \lambda k\{C(n)-A(n)\}-k\{Q(n)-P(n)\} .
$$

3. Es sollen nun die rechten Seiten von (2.1) und (2.2) nach unten abgeschätzt werden. Wir setzen

$$
\begin{aligned}
\Gamma(k) & \left.=\sum_{m=1}^{n-k-1} P(m)\{Q(m+k+1)-Q(m+k)\}^{4}\right), \\
\Delta(n) & =\sum_{m=1}^{n} E(m)
\end{aligned}
$$

und entsprechend

$$
\begin{aligned}
& \bar{\Gamma}(k)=\sum_{m=1}^{n-k-1} \bar{P}(m)\{\bar{Q}(m+k+1)-\bar{Q}(m+k)\}, \\
& \bar{\Delta}(n)=\sum_{m=1}^{n} \bar{E}(m) .
\end{aligned}
$$

4) Eine Summe, bei der die untere Summationsgrenze größer als die obere ist, sei gleich Null. (Dies ist hier für $k=n-1$ und $k=n$ der Fall.) 
Hilfssatz 2. Es gelten die folgenden Beziehungen:

$$
\begin{aligned}
& \sum_{m=1}^{k} E(m)=\Delta(n)-\Gamma(k) \leqq\left(\begin{array}{c}
P(n) \\
2
\end{array}\right)+\{Q(n)-P(n)\} P(n)-\Gamma(k) \\
& \sum_{m=1}^{k} \bar{E}(m)=\bar{\Delta}(n)-\bar{\Gamma}(k) \leqq\left(\begin{array}{c}
\bar{Q}(n) \\
2
\end{array}\right)+\{\bar{P}(n)-\bar{Q}(n)\} \bar{Q}(n)-\bar{\Gamma}(k)
\end{aligned}
$$

Beweis. Wir beweisen zunächst die in (2.10) und (2.11) links stehenden Gleichungen. Für $k=n-1$ und $k=n$ sind diese offenbar erfüllt. Für $k \leqq n-2$ ist zum Beweis der in (2.10) auftretenden Gleichung offenbar

$$
\sum_{m=k+1}^{n} E(m)=\Gamma(k)=\sum_{m=1}^{n-k-1} P(m)\{Q(m+k+1)-Q(m+k)\}
$$

nachzuweisen. Nach Definition von $E(m)$ werden in der links stehenden Summe alle verschiedenen Paare $(p, q)$ mit $p \in \mathfrak{P}(n), q \in \mathfrak{D}(n)$ und $q-p \geqq k+1$ gezählt. Die Anzahl dieser Paare ist bei festem $q$ offenbar gleich $P(q-k-1)$. Durch Summation über alle $q \in \mathfrak{D}(n)$ mit $q \geq k+2$ (denn nur solche $q$ kommen wegen $p>0$ in Frage) erhält man

Wegen

$$
\sum_{m=k+1}^{n} E(m)=\sum_{\substack{q \in \mathfrak{Q}(n) \\ q \geq k+2}} P(q-k-1) .
$$

folgt daraus

$$
Q(m)-Q(m-1)=\left\{\begin{array}{l}
1 \text { falls } m \in \mathfrak{} \\
0 \text { falls } m \notin \Omega
\end{array}\right.
$$

$$
\begin{aligned}
\sum_{m=k+1}^{n} E(m) & =\sum_{m=k+2}^{n} P(m-k-1)\{Q(m)-Q(m-1)\} \\
& =\sum_{m=1}^{n-k-1} P(m)\{Q(m+k+1)-Q(m+k)\}=\Gamma(k),
\end{aligned}
$$

womit (2.12) bewiesen ist. Die gleiche Überlegung für die komplementären Mengen liefert die in (2.11) stehende Gleichung.

Zum Beweis von (2.10) und (2.11) sind noch die Abschätzungen

$$
\begin{aligned}
& \Delta(n)=\sum_{m=1}^{n} E(m) \leqq\left(\begin{array}{c}
P(n) \\
2
\end{array}\right)+\{Q(n)-P(n)\} P(n), \\
& \bar{\Delta}(n)=\sum_{m=1}^{n} \bar{E}(m) \leqq\left(\begin{array}{c}
\bar{Q}(n) \\
2
\end{array}\right)+\{\bar{P}(n)-\bar{Q}(n)\} \bar{Q}(n)
\end{aligned}
$$

nachzuweisen. In $\sum_{m=1}^{n} E(m)$ werden alle verschiedenen Paare $(p, q)$ mit $p \in \mathfrak{P}(n)$, $q \in \Omega(n)$ und $q-p>0$ gezählt. Wegen $\mathfrak{\beta} \leqq \Omega$ werden dabei auch alle Paare $\left(p, p^{\prime}\right)$ mit $p, p^{\prime} \in \mathfrak{B}(n)$ und $p^{\prime}-p>0$ gezählt, und diese Anzahl ist gleich $\left(\begin{array}{c}P(n) \\ 2\end{array}\right)$. Es bleibt dann noch die Anzahl der Paare $(p, q)$ mit $p \in \mathfrak{F}(n)$, 
$q \in \Omega(n)$, aber $q \notin$ 壮 $(n)$ und $q-p>0$ abzuschätzen. Läßt man die Forderung $q-p>0$ fallen, so ist diese Anzahl genau gleich $\{Q(n)-P(n)\} P(n)$. Damit ist (2.13) bewiesen. Zum Beweis von (2.14) beachte man $\bar{D} \leqq \overline{P^{3}}$ und schließe sinngemäß.

4. Aus (2.1), (2.5) und (2.10) erhält man die folgende

1. Grundformel $\left.{ }^{5}\right)$ :

$$
\left\{\begin{array}{l}
\lambda k\{C(n)-A(n)\}-k\{Q(n)-P(n)\} \geqq \sum_{m=n-k}^{n-1} P(m)+ \\
+\sum_{m=1}^{n-k-1} P(m)\{Q(m+k+1)-Q(m+k)\}-\left(\begin{array}{c}
P(n) \\
2
\end{array}\right)-\{Q(n)-P(n)\} P(n) .
\end{array}\right.
$$

Entsprechend folgt aus (2.2), (2.6) und (2.11) die

2. Grundformel:

$$
\left\{\begin{array}{l}
\lambda k\{C(n)-A(n)\}-k\{Q(n)-P(n)\} \geqq k \bar{Q}(n)-\sum_{m=1}^{k} \bar{Q}(m)+ \\
+\sum_{m=1}^{n-k-1} \bar{P}(m)\{\bar{Q}(m+k+1)-\bar{Q}(m+k)\}-\left(\begin{array}{c}
\bar{Q}(n) \\
2
\end{array}\right)-\{\bar{P}(n)-\bar{Q}(n)\} \bar{Q}(n) .
\end{array}\right.
$$

Für $\mathfrak{P}=\mathfrak{Q}=\mathfrak{A}$ erhält man daraus die Grundformeln aus (I). Im folgenden sollen die Grundformeln in den Spezialfällen $\mathfrak{P}=\mathfrak{A}$, $\mathfrak{D}=\mathfrak{C}$ und $\mathbb{*}=\mathfrak{\Omega}=\mathbb{C}$ ausgewertet werden.

\section{§3. Auswertung der 1. Grundformel für $\mathfrak{W}=\mathfrak{i}, \grave{\Sigma}=\mathfrak{C}$.}

1. Das Ziel dieses Paragraphen ist der Beweis der folgenden Abschätzungen: Finiter Fall. Setzt man

$$
c_{1}(\alpha)=\frac{1+\sqrt{\alpha}+\alpha}{(1+\sqrt{\alpha})^{2}}, \quad c_{3}(\alpha, \lambda)=c_{1}(\alpha) \frac{\lambda}{\lambda-1+(1+\alpha) / \alpha},
$$

dann gilt

$$
\left.\frac{C(n)}{n}>\alpha\left(1+c_{3}(\alpha, \lambda) \frac{1-\alpha}{\lambda}\right)^{6}\right) .
$$

Asymptotischer Fall. Besteht mit den durch (3.1) gegebenen Funktionen die Ungleichung

$$
\left(\lambda^{*}-1\right) \sqrt{\alpha^{*}}+\alpha^{*}\left\{\frac{3}{2}\left(1+\alpha^{*}\right)-\sqrt{\alpha^{*}}\right\}>\alpha^{*}\left(1+\alpha^{*}\right)\left(1+c_{3}\left(\alpha^{*}, \lambda^{*}\right) \frac{1-\alpha^{*}}{\lambda^{*}}\right)
$$

dann gilt

$$
\gamma^{*} \geqq \alpha^{*}\left(1+c_{3}\left(\alpha^{*}, \lambda^{*}\right) \frac{1-\alpha^{*}}{\lambda^{*}}\right) .
$$

Die Voraussetzung (3.3), die im finiten Fall tehlt, stellt keine wesentliche Einschränkung dar, denn z.B. für $\lambda^{*} \geqq_{\frac{3}{2}}^{3}$ ist sie bereits identisch in $\alpha^{*}$

5) Man beachte Fußnote 4 auf S. 245.

$\left.{ }^{6}\right)$ In (I) wurde diese Ungleichung mit $c_{1}(\alpha)$ an Stelle von $c_{3}(\alpha, \lambda)$ hergeleitet. 
erfüllt, und $\mathrm{zu}$ jedem kleineren $\lambda^{*}$ gibt es eine positive Zahl $\alpha_{\mathbf{0}}^{*}$, so da13 (3.3) für alle $\alpha^{*}<\alpha_{0}^{*}$ richtig ist.

Das Wesentliche an $c_{3}(\alpha, \lambda)$ ist die Tatsache, daß

$$
\lim _{\alpha \rightarrow 0} c_{3}(\alpha, \lambda)=\frac{\lambda}{\lambda-1}
$$

gilt. Daraus und aus der vorhergehenden Bemerkung folgt, daß es zu jedem $\lambda$ bzw. $\lambda^{*}$ positive Zahlen $\alpha_{\lambda}$ bzw. $\alpha_{\lambda^{*}}^{*}$ gibt, so daß für alle $\alpha<\alpha_{i}$ bzw. $\alpha^{*}<\alpha_{\lambda^{*}}^{*}$ die ERDössche Vermutung (1.1) bzw. die entsprechend asymptotische Ungleichung übertroffen werden.

In der Abbildung in $(\mathrm{I})$ ist $c_{3}(\alpha, \lambda)$ für $\lambda=\underset{6}{19}$ eingetragen. In diesem Falle kann $\alpha_{\lambda}>\frac{1}{9}$ gewählt werden.

2. Als Hilfsmittel zum Beweis leiten wir jetzt einen Reduktionssatz her, der auch an sich von Interesse ist.

Reduktionssatz. Es sei $\varphi(\alpha)$ eine für $0 \leqq \alpha \leqq 1$ monoton wachsende Funktion von $\alpha$, und es sei $\mathfrak{B}$ eine die 0 enthaltende Menge nichtnegativer ganzer Zahlen. Gilt dann für alle Mengen nichtnegativer ganzer Zahlen $\mathfrak{A}$ mit $1 \in \mathfrak{A}$, und alle natürlichen Zahlen $n$ mit $\operatorname{Min}_{i=1, \ldots, n} \frac{A(i)}{i}=\frac{A(n)}{n}$ die. Ungleichung

$$
(A+B)(n)>\varphi(\alpha) n,
$$

wobei $(A+B)(n)$ die Anzahlfunktion der Summenmenge $\mathfrak{A}+\mathfrak{B}$ und $\alpha$ die Dichte von $\mathfrak{A}$ seien, dann gilt diese Ungleichung fïr alle natïrlichen Zahlen $n^{7}$ ).

Beweis. Induktion nach $n$. Für $n=1$ gilt die Behauptung nach Voraussetzung; sie sei bis $n-1$ bewiesen. Sei $\mathfrak{A}$ eine beliebige Menge mit $1 \in \mathfrak{A}$, dann können wir $\operatorname{Min}_{i=1, \ldots, n} \frac{A(i)}{i}<\frac{A(n)}{n}$ annehmen, da die Behauptung sonst nach Voraussetzung gilt. Wird das Minimum für $i=m_{0}$ angenommen, so ist folglich $m_{0}<n$. Ferner können wir ohne Einschränkung $\frac{A\left(m_{0}\right)}{m_{0}}=\alpha$ annehmen, da dies sonst durch Auffüllen der Menge $\mathfrak{U}$ von der Zahl $n+1$ ab erreicht werden kann; dies ändert nichts an $(A+B)(n)$, und die Dichte von $\mathfrak{A}$ wird dabei höchstens vergrößert. Insbesondere gilt dann $\alpha>0$. Wir betrachten jetzt die Menge $\mathfrak{A}_{0}=\left\{a-m_{0}\right\}$ mit $a \in \mathfrak{U}, a>m_{0}$. Für diese gilt

$$
A_{0}(r)=A\left(r+m_{0}\right)-A\left(m_{0}\right) \geqq\left(r+m_{0}\right) \alpha-m_{0} \alpha=r \alpha, \quad(r=1,2, \ldots),
$$

also $\alpha_{0} \geqq \alpha>0$, wenn $\alpha_{0}$ die Dichte von $\mathfrak{A}_{0}$ bezeichne. Für $r=1$ folgt $1 \in \mathfrak{A}_{0}$. Nach Induktionsvoraussetzung hat man daher

$$
\left(A_{0}+B\right)\left(n-m_{0}\right)>\varphi\left(\alpha_{0}\right)\left(n-m_{0}\right) \geqq \varphi(\alpha)\left(n-m_{0}\right) .
$$

7) Der Satz gilt auch, wenn man nicht $1 \in \mathfrak{U}$ und nur $(A+B)(n) \sum \varphi(\alpha) n$ fordert. Der Beweisgedanke für diesen Satz findet sich in anderer Form bereits bei D. RaIKov [5] (beim Beweis des Analogon zum $\alpha-\beta$-Theorem), wo Dichtesätze für Mengen reeller Zahlen bewiesen werden. - Es sei darauf hingewiesen, daß sich die Basisabschätzungen in $[\tilde{j}]$. bei denen der Beweis von ERDös-Landau [4] in die mengentheoretische Sprechweise übertragen wird, durch Übertragung unserer Überlegungen in analoger Weise verschärfen lassen.

Zusatz bei der Korrektur: Wie mir Herr E. Wirsing mitteilte, war ihm vorstehender Reduktionssatz auch bekannt. 
Liegt eine natürliche Zahl $r \leqq n-m_{0}$ in $\mathfrak{A}_{0}+\mathfrak{b}$, so liegt $r+m_{0}$ in $\mathfrak{A}+\mathfrak{B}$, und zwar im Intervall $m_{0}<r+m_{0} \leqq n$; also gilt

$$
\left(A_{0}+B\right)\left(n-m_{0}\right) \leqq(A+B)(n)-(A+B)\left(m_{0}\right) .
$$

Die beiden letzten Ungleichungen zusammen liefern die Behauptung.

Wir weisen darauf hin, daß auf Grund dieses Satzes die Beweise der Abschätzungen in (I) im finiten Fall vereinfacht werden können, da dadurch die Minimalbetrachtung [(I), S. 377] beim Beweis von (I.1.8) und die Untersuchung des Koeffizienten von $A(n)$ [(I), S. 383] beim Beweis von (I.1.9) überflüssig werden.

3. Zum Beweis von (3.2) gehen wir von der 1. Grundformel (2.15) aus, in der wir $\mathfrak{P}=\mathfrak{A}$ und $\mathfrak{Q}=\mathfrak{C}$ setzen:

(3.6)

$$
\left\{\begin{array}{l}
(\lambda-1) k\{C(n)-A(n)\} \geqq \sum_{m=n-k}^{n-1} A(m)+\sum_{m=1}^{n-k-1} A(m)\{C(m+k+1)-C(m+k)\}- \\
\quad-\left(\begin{array}{c}
A(n) \\
2
\end{array}\right)-\{C(n)-A(n)\} A(n) .
\end{array}\right.
$$

Wegen $\mathfrak{U} \leqq(5$ gilt $C(m+k+1)-C(m+k) \geqq A(m+k+1)-A(m+k)$, so da $B$ aus (3.6) folgt:

$$
\left\{\begin{array}{l}
(\lambda-1) k\{C(n)-A(n)\} \geqq \sum_{m=n-k}^{n-1} A(m)+\sum_{m=1}^{n-k-1} A(m)\{A(m+k+1)-A(m+k)\}- \\
-\left(\begin{array}{c}
A(n) \\
2
\end{array}\right)-\{C(n)-A(n)\} A(n) .
\end{array}\right.
$$

Bis auf den Summanden $-\{C(n)-A(n)\} A(n)$ stimmt die rechte Seite dieser Ungleichung mit der rechten Seite von (I.3.1) überein; links steht jetzt jedoch der Faktor $\lambda-1$ an Stelle von $\lambda$ in (I.3.1). Wir machen jetzt die Voraussetzung $k \leqq n-2$ und können dann die auf (I.3.1) folgende Schlußweise unter gewissen Einschränkungen für den vorliegenden Fall übernehmen. Einer erneuten Rechtfertigung bedürfen die dort im Anschluß an (I.3.5) gemachten Voraussetzungen.

Zunächst kann auch jetzt $\alpha \leqq \frac{1}{2}$ angenommen werden, da sonst bereits $c_{1}(\alpha)$ besser als $c_{3}(\alpha, \lambda)$ ist. Ferner kann $n \geq 4$ angenommen werden, wic jetzt gezeigt werden soll. Ist $C(n)=n$, so folgt (3.2) unmittelbar aus der Tatsache, daß

$$
\alpha\left(1+c_{3}(\alpha, \lambda) \frac{1-\alpha}{\lambda}\right)<\alpha+\sqrt{\alpha}(1-\alpha)<1
$$

gilt. Dies ist wegen $1 \in \mathfrak{A}$ und $1 \in \mathfrak{B}$ für $n=1$ und $n=2$ der Fall, sowie für $n=3$, wenn $\mathfrak{A}(3) \neq\{1\}$ ist. Sei nun $\mathfrak{A}(3)=\{1\}$; dann ist $\alpha \leq \frac{1}{3}$. Für $\lambda<\frac{3}{2}$ müssen die Zahlen 1 und 2 in $\mathfrak{B}$ enthalten sein, so daß auch jetzt $C(3)=3$ folgt. Sei also $\lambda \geqq \frac{3}{2}$; dann gilt

$$
\begin{aligned}
\frac{C(3)}{3} & \geq \frac{2}{3} \geq \alpha+\frac{1}{3}>\alpha+\frac{\frac{1}{3}\left(1-\frac{1}{3}\right)}{\frac{1}{2}+\frac{1}{3}} \\
& \geq \alpha\left(1+\frac{1-\alpha}{\lambda-1+\alpha}\right)>\alpha\left(1+c_{3}(\alpha, \lambda) \frac{1-\alpha}{\lambda}\right) .
\end{aligned}
$$

Es darf also jetzt $n \geq 4$ vorausgesetzt werden. 
Ferner kann auch jetzt $\varrho n \geq 1$ angenommen werden. Ist $\varrho n=\sqrt{\alpha}(1+\sqrt{\alpha})^{-1} n$ $<1$, so hat man wegen $n \geqq 4$ die Schranke $\alpha<\frac{1}{11}$. Es ist dann zunächst

kann die Ungleichung

$$
\frac{C(n)}{n} \geqq \frac{2}{n}>\frac{2 \gamma^{\prime \alpha}}{1+\gamma^{\alpha} \alpha}
$$

$$
\frac{2 \sqrt{\alpha}}{1+\gamma / \alpha} \geqq \alpha\left(1+c_{3}(\alpha, \lambda) \frac{1-\alpha}{\lambda}\right)
$$

bewiesen werden, so ist man fertig. Diese ist richtig, falls

gilt, denn es ist

$$
\frac{2 \sqrt{\alpha}}{1+\sqrt{\alpha}} \geqq \sqrt{\alpha}(\sqrt{\alpha}+1)
$$

$$
\sqrt{\alpha}(\sqrt{\alpha}+1)=\alpha+\sqrt{\alpha} \geqq \alpha+\frac{\alpha(1-\alpha)}{(1+\alpha) \sqrt{\alpha}} \geqq \alpha\left(1+c_{3}(\alpha, \lambda) \frac{1-\alpha}{\lambda}\right) .
$$

Die Ungleichung (3.8) gilt aber wegen $\alpha<\frac{1}{y}$.

Wir können nun ebenso wie im Anschluß an (I.3.5) weiterschließen und erhalten in Analogie zu (I.3.7)

$$
\begin{aligned}
& (\lambda-1)\{(1-\alpha) \varrho+\alpha\} \frac{C(n)}{n}>((\lambda-1)\{(1-\alpha) \varrho+\alpha\}+\alpha\{1-(1-\alpha) \varrho\}) \frac{A(n)}{n}- \\
& -\frac{1+\alpha}{2} \cdot \frac{A^{2}(n)}{n^{2}}+\frac{\left(1-\alpha^{2}\right) \alpha}{2} \varrho(2-\varrho)-(1+\alpha)\{C(n)-A(n)\} \frac{A(n)}{n^{2}} .
\end{aligned}
$$

Setzt man darin $\varrho=\frac{1 / \alpha}{1+\gamma^{\alpha}}$ ein, so folgt

$$
\left\{\begin{array}{c}
(\lambda-1) \sqrt{\alpha-\frac{C(n)}{n}}>\left((\lambda-1) / \alpha+\alpha\left\{\frac{3}{2}(1+\alpha)-\sqrt{\alpha}\right\}-(1+\alpha) \frac{C(n)}{n}\right) \times \\
\times \frac{A(n)}{n}+\frac{\left(1-\alpha^{2}\right) \alpha}{2} \frac{\sqrt{\alpha}(1-\mid \alpha)\left(2-\alpha-\gamma^{\prime} \alpha\right)}{(1-\alpha)^{2}} .
\end{array}\right.
$$

Auf Grund des Reduktionssatzes können wir darin $\frac{A(n)}{n}$ durch $\alpha$ ersetzen und dann ergibt sich unmittelbar (3.2).

Im asymptotischen Fall erhält man durch eine entsprechende Überlegung eine Formel, die aus (3.9) hervorgeht, wenn man darin überall $\alpha$ durch $\alpha^{\prime}=\alpha-\varepsilon, \lambda$ durch $\lambda^{\prime}=\lambda+\mu$ ersetzt und ein Restglied $K / n$ auf der recliten Seite addiert. In dieser Formel darf $\frac{A(n)}{n}$ durch $\alpha^{\prime}$ ersetzt werden, wenn der Koeffizient von $\frac{A(n)}{n}$ nicht negativ ist. Ist dieser Koeffizient jedoch negativ, so sichert Voraussetzung (3.3) unsere Behauptung; dabei hat man zu berücksichtigen, da $\beta \varepsilon$ und $\mu$ so klein gewählt werden können, da $\beta$ auch für die gestrichenen Größen auf der linken Seite von (3.3) diese Ungleichung richtig bleibt. Durch Grenzübergang folgt schließlich (3.4).

Es soll darauf hingewiesen werden, da $\beta c_{3}(\alpha, \lambda)$ [bzw. $\left.c_{3}\left(\alpha^{*}, \lambda^{*}\right)\right]$ nicht der beste Faktor ist, den man auf diesem Wege herleiten kann. Eine Verbesserung läßt sich erreichen, indem man $\varrho$ nicht nur in Abhängigkeit von $\alpha$, sondern 
auch noch von $\lambda$ (und eventuell $\gamma$ ) wählt. Das führt jedoch zu sehr unübersichtlichen Formeln; der so zu gewinnende Faktor hat außerdem auch nur wieder die Eigenschaft, für $\alpha \rightarrow 0$ gegen $\frac{\lambda}{\lambda-1}$ zu streben. Wir wollen daher nicht weiter auf diese Möglichkeit eingehen.

\section{$\S 4$. Auswertung der Grundformeln für $=\mathfrak{D}=\boldsymbol{C}$.}

1. Wie schon erwähnt, erhält man die Grundformeln in (I) aus (2.15) und (2.16) für $\mathfrak{P}=\mathfrak{\Omega}=\mathfrak{A}$. Wir betrachten hiẹr die aus (2.15) und (2.16) für $\mathfrak{B}=\mathfrak{Q}=\mathbb{C}$ folgenden Abschätzungen, bei denen auf der rechten Seite statt $A$ wie in (I) jetzt überall $C$ steht:

$$
\begin{gathered}
\left\{\begin{array}{c}
\lambda k\{C(n)-A(n)\} \geqq \sum_{m=n-k}^{n-1} C(m)+\sum_{m=1}^{n-k-1} C(m)\{C(m+k+1)- \\
-C(m+k)\}-\left(\begin{array}{c}
C(n) \\
2
\end{array}\right)
\end{array}\right. \\
\left\{\begin{array}{c}
\lambda k\{C(n)-A(n)\} \geqq k \bar{C}(n)-\sum_{m=1}^{k} \bar{C}(m)+\sum_{m=1}^{n-k-1} \bar{C}(m)\{\bar{C}(m+k+1)- \\
-\bar{C}(m+k)\}-\left(\begin{array}{c}
\bar{C}(n) \\
2
\end{array}\right) .
\end{array}\right.
\end{gathered}
$$

Während in (I) aus den Grundformeln die Abschätzungen

$$
\frac{C(n)}{n}>\alpha+c_{1}(\alpha) \frac{\alpha(1-\alpha)}{\lambda}, \quad \frac{C(n)}{n}>\alpha+c_{1}(1-\alpha) \frac{\alpha(1-\alpha)}{\lambda}
$$

hergeleitet worden waren, erhält man jetzt aus (4.1) und (4.2) die folgenden Abschätzungen, die auch an sich von Interesse sind:

$$
\begin{aligned}
& \frac{C(n)}{n}>\alpha+c_{1}(\gamma) \frac{\gamma(1-\gamma)}{\lambda}, \\
& \frac{C(n)}{n}>\alpha+c_{1}(1-\gamma) \frac{\gamma(1-\gamma)}{\lambda} .
\end{aligned}
$$

Entsprechend erhält man im asymptotischen Fall:

$$
\begin{aligned}
& \gamma^{*} \geqq \alpha^{*}+c_{1}\left(\gamma^{*}\right) \frac{\gamma^{*}\left(1-\gamma^{*}\right)}{\lambda^{*}}, \\
& \left.\gamma^{*} \geqq \alpha^{*}+c_{1}\left(1-\gamma^{*}\right) \frac{\gamma^{*}\left(1-\gamma^{*}\right)}{\lambda^{*}}{ }^{8}\right) .
\end{aligned}
$$

^) Bei der Andeutung des Beweises der entsprechenden Formel in (I) (Beweis von $c_{2}^{*}$, S. 384), habe ich irrtümlich geschrieben, daß die Schnirelmannsche Ungleichung auch im asymptotischen Falle gelte. Das ist bekanntlich im allgemeinen nicht der Fall und wird auch gar nicht benutzt. Tatsächlich gebraucht wird sie in (I) nur für $\alpha^{*}>\frac{1}{2}$ und $\beta^{*} \geq \frac{\frac{2}{2} 5}{2}$ (also $\lambda^{*} \leq \frac{29}{2} \frac{9}{7}$ ), da die Behauptung sonst unmittelbar aus der zu (I.4.10) analogen asymptotischen Formel folgt. Aber bereits für $\alpha^{*}>\frac{1}{2}$ und $\beta^{*} \geq \frac{t}{2}$ ergibt sich ihre Gültigkeit aus dem Dichtesatz von M. KNESER [3] (oder auch aus schwächeren Abschätzungen). - Bei dieser Gelegenheit sei noch auf zwei Druckfehler in (I) hingewiesen; auf S. 370, 7. Zeile v. u. muß es ,H. Rohrbach [8]" (statt [10]) und auf S. 376, 3. Zeile v. u. ,A. StöHr [11]“" (statt [13]) lauten. 
Die Beweise können - abgesehen von einer Vereinfachung - fast unmittelbar aus (I) $(\S \S 3,4)$ übernommen werden; man hat dort auf der rechten Seite der Abschätzungen nur überall $A(n), \alpha, \ldots$ durch die entsprechenden Größen $C(n), \gamma, \ldots$ zu ersetzen. Man muß sich lediglich klarmachen, da $\beta$ die dort für $A(n), \alpha, \ldots$ gemachten Voraussetzungen auch für $C(n), \gamma, \ldots$ gelten. Das soll jetzt erfolgen.

Wir betrachten zunächst den finiten Fall. Wegen $\alpha<1$ und $1 \in \mathfrak{B}$ gilt stets $\frac{C(n)}{n}>\alpha$; (4.3) und (4.4) sind also für $\gamma=1$ erfüllt, so da $\beta$ analog zur Voraussetzung über $\alpha$ in (I) jetzt $0<\gamma<1$ vorausgesetzt werden kann. Darüber hinaus kann jetzt noch die zusätzliche Voraussetzung gemacht werden, daß $\gamma$ für einen endlichen Wert $n$ von $\frac{C(n)}{n}$ angenommen wird. Ist dies nämlich nicht der Fall, dann gilt offenbar $\gamma=\gamma^{*}$ und $\frac{C(n)}{n}>\gamma(n=1,2, \ldots)$; die finiten Abschätzungen (4.3) und (4.4) folgen dann wegen $\alpha^{*} \geqq \alpha$ und $\lambda^{*} \leq \lambda$ aus den asymptotischen Abschätzungen (4.5) und (4.6).

Die Ungleichungen (4.1) und (4.2) werden nun für eine Zahl $n$ mit $\frac{C(n)}{n}=\gamma$ analog zur Schlußweise in (I, §§3,4) abgeschätzt, wobei allerdings jetzt keine Minimumbetrachtungen notwendig sind, die, wie im Anschluß an den Reduktionssatz schon erwähnt, auch in (I) wegfallen können.

Entsprechend schließt man auch im asymptotischen Fall. So wird z.B. die auf (I.3.11) folgende Minimumbetrachtung jetzt durch die folgende Überlegung ersetzt. Nach Definition von $\gamma^{*}$ gibt es eine unendliche Folge von natürlichen Zahlen $n$ mit $\frac{C(n)}{n} \rightarrow \gamma^{*}$, die dann zu vorgegebenem $\varepsilon>0$ für hinreichend großes $n$

$$
\gamma^{\prime}=\gamma^{*}-\varepsilon \leqq \frac{C(n)}{n} \leqq \gamma^{*}+\varepsilon=\gamma^{\prime \prime}
$$

genügen. In der (I.3.11) entsprechenden Ungleichung

$$
\left\{\begin{array}{r}
\lambda^{\prime}\left\{\varrho+\gamma^{\prime}(1-\varrho)\right\} \frac{C(n)-A(n)}{n} \geq \gamma^{\prime}\left\{1-\left(1-\gamma^{\prime}\right) \varrho\right\} \frac{C(n)}{n}- \\
-\frac{1+\gamma^{\prime}}{2} \frac{C^{2}(n)}{n^{2}}+\frac{\left(1-\gamma^{\prime 2}\right) \gamma^{\prime}}{2} \varrho(2-\varrho)+K \frac{1}{n}
\end{array}\right.
$$

und bei dem folgenden Grenzübergang $n \rightarrow \infty$ seien nur solche Zahlen $n$ berücksichtigt. Aus (4.7) folgt dann

$$
\begin{aligned}
\lambda^{\prime}\{\varrho & \left.+\gamma^{\prime}(1-\varrho)\right\}\left(\gamma^{\prime \prime}-\alpha^{\prime}\right) \geqq\left\{1-\left(1-\gamma^{\prime}\right) \varrho\right\} \gamma^{\prime 2}-\frac{1+\gamma^{\prime}}{2} \gamma^{\prime \prime 2}+ \\
& +\frac{\left(1-\gamma^{\prime 2}\right) \gamma^{\prime}}{2} \varrho(2-\varrho)+K \frac{1}{n} .
\end{aligned}
$$

Setzt man $\varrho=\frac{\sqrt{\gamma^{\prime}}}{1+\gamma \gamma^{\prime}}$ ein, so erhält man

$$
\gamma^{\prime \prime} \geq \alpha^{\prime}+\frac{1+\sqrt{\gamma^{\prime}}+\gamma^{\prime}}{\left(1+\sqrt{\gamma^{\prime}}\right)^{2}} \frac{\gamma^{\prime}\left(1-\gamma^{\prime}\right)}{\lambda^{\prime}}-\frac{1+\gamma^{\prime}}{2 \lambda^{\prime} /\left(\gamma^{\prime}\right.} \gamma^{*} \varepsilon+K \frac{1}{n},
$$

woraus (4.5) folgt. 
2. Aus (4.3) und (4.5) können wieder Abschätzungen von $\gamma$ bzw. $\gamma^{*}$ durch Ausdrücke in $\alpha$ bzw. $\alpha^{*}$ hergeleitet werden. Es seien

$$
\begin{aligned}
c_{1}(x) & =\frac{1+\sqrt{x}+x}{(1+\sqrt{x})^{2}}, \quad \sigma(x)=x\left(1+c_{1}(x) \frac{1-x}{\lambda-c_{1}(x)}\right), \quad c_{0}(x)=c_{1}(\sigma(x)) \\
\nu(x) & =\frac{1}{2 c_{0}(x)}\left\{\sqrt{\left\{\lambda-(1-2 x) c_{0}(x)\right\}^{2}+4 x(1-x) c_{0}^{2}(x)}-\left\{\lambda-(1-2 x) c_{0}(x)\right\},\right. \\
c_{4}(x) & =c_{4}(x, \lambda)=c_{0}(x) \frac{\lambda}{\lambda-c_{0}(x)\{1-2 x-\nu(x)\}} .
\end{aligned}
$$

Wegen $\lim _{x \rightarrow 0} \sigma(x)=0$ gilt $\lim _{x \rightarrow 0} c_{0}(x)=1$; daraus folgt $\lim _{x \rightarrow 0} v(x)=0$ sowie

$$
\lim _{x \rightarrow 0} c_{4}(x)=\frac{\lambda}{\lambda-1} .
$$

Mit diesen Funktionen gelten die folgenden Ungleichungen:

$$
\begin{gathered}
\gamma>\alpha\left(1+c_{4}(\alpha, \lambda) \frac{1-\alpha}{\lambda}\right), \\
\gamma^{*}>\alpha^{*}\left(1+c_{4}\left(\alpha^{*}, \lambda^{*}\right) \frac{1-\alpha^{*}}{\lambda^{*}}\right) .
\end{gathered}
$$

Es ist klar, daß die Ungleichungen (4.9) und (4.10) richtig bleiben, wenn man $v(\alpha)$ bzw. $v\left(\alpha^{*}\right)$ durch größere Zahlen ersetzt. Zum Beispiel gelten die Ungleichungen

$$
\nu(\alpha) \leqq c_{1}(\alpha) \frac{\alpha(1-\alpha)}{\lambda-c_{1}(\alpha)(1-2 \alpha)}, \quad v(\alpha) \leqq \sqrt{\alpha(1-\alpha)},
$$

aus denen bereits (4.8) folgt.

Die Beweise von (4.9) und (4.10) sollen nicht im einzelnen ausgeführt werden. Wir beschränken uns auf folgenden Hinweis: Zum Beweis von (4.9) gehe man von (4.3) in der Form

$$
\gamma\left(1-c_{1}(\gamma) \frac{1-\gamma}{\lambda}\right) \geqq \alpha
$$

aus, wobei die linke Seite eine monoton wachsende Funktion von $\gamma$ ist; nimmt man an, daß (4.9) falsch sei, so kann man in (4.11) $\gamma$ durch $\alpha\left(1+c_{4} \frac{1-\alpha}{\lambda}\right)$ ersetzen, was durch einige Rechnung zum Widerspruch führt.

\section{$\S 5$. Abschätzung der Anzahlfunktion einer Summenmenge von Gitterpunkten.}

Es liegt die Frage nahe, ob man mit den Methoden der additiven Zahlentheorie auch additive Eigenschaften von Gitterpunktmengen in einem endlichdimensionalen Raum untersuchen kann. Im folgenden soll gezeigt werden. da $\beta$ diese Frage für das in dieser Arbeit behandelte Problem zu bejahen ist. Wir wollen uns darauf beschränken, die ERDös-LANDAusche Ungleichung $\gamma \geqq \alpha\left(1+\frac{1-\alpha}{2 \lambda}\right)$ in diesem Sinne zu verallgemeinern. 
Unter einem Gitterpunkt des $r$-dimensionalen Raums verstehen wir einen Punkt mit ganzrationalen Koordinaten, den wir in der Form

$$
\mathfrak{r}=\left(n_{1}, \ldots, n_{r}\right) \quad\left(n_{\imath} \operatorname{gan} z\right)
$$

schreiben. Es sei $\mathfrak{B}$ die Menge der Gitterpunkte mit nichtnegativen Koordinaten und $\mathfrak{N}$ die Menge aller vom Nullpunkt verschiedenen Gitterpunkte aus $\mathfrak{B}$. Die Gitterpunkte aus $\mathfrak{N}$ wollen wir als natürliche Gitterpunkte bezeichnen.

Es seien $\mathfrak{A}, \mathfrak{B}, \mathfrak{C}, \ldots$ Untermengen von $\mathfrak{B}$. Unter $\mathfrak{A}(\mathfrak{n}), \mathfrak{n}=\left(n_{1}, \ldots, n_{r}\right) \in \mathbb{2}$ verstehen wir die Menge aller $\mathfrak{a}=\left(a_{1}, \ldots, a_{r}\right) \in \mathfrak{U}$ mit $a_{i} \leqq n_{i}(i=1, \ldots, r)$. Die Anzahlfunktion $A(\mathfrak{n})$ (mit $\mathfrak{n} \in \mathfrak{N}$ ) der Menge $\mathfrak{U}$ sei die Anzahl der natürlichen Gitterpunkte aus $\mathfrak{A}(\mathfrak{n})$. In Analogie zur Definition im linearen Fall wird

$$
\underset{\mathfrak{n} \in \mathfrak{\Omega}}{\operatorname{fin}} \frac{A(\mathfrak{n})}{N(\mathfrak{n})}=\alpha
$$

als (finite) Dichte von $\mathfrak{A}$ bezeichnet $^{9}$ ). Dann gilt für jeden natürlichen Gitterpunkt $\mathfrak{n}: A(\mathfrak{n}) \geqq \alpha N(\mathfrak{n})$.

Wir setzen im folgenden $\mathfrak{A} \leqq \Re$ und $0<\alpha<1$ voraus. Aus $0<\alpha$ folgt, daß die Einheitspunkte, d.h. die Punkte

$$
(1,0, \ldots, 0),(0,1,0, \ldots, 0), \ldots,(0, \ldots, 0,1)
$$

in $\mathfrak{A}$ enthalten sind, und aus $\alpha<1$ folgt, daß $\mathfrak{A}$ nicht alle natürlichen Gitterpunkte enthält.

Sei $\overline{\mathfrak{A}}$ die Komplementärmenge von $\mathfrak{A}$ in $\mathfrak{R}$ mit der Anzahlfunktion $\bar{A}(\mathfrak{n})$, dann hat man offenbar $\bar{A}(\mathfrak{n})=N(\mathfrak{n})-A(\mathfrak{n})$, und es gilt $\bar{A}(\mathfrak{n}) \leqq(1-\alpha) N(\mathfrak{n})$.

Unter der Summenmenge $\mathfrak{C}=\mathfrak{A}+\mathfrak{B}$ mit der Dichte $\gamma$ und der Anzahlfunktion $C(\mathfrak{n})$ ist die Menge aller in der Gestalt

$$
\mathfrak{a}+\mathfrak{b}=\left(a_{1}+b_{1}, \ldots, a_{r}+b_{r}\right)
$$

darstellbaren Gitterpunkte mit $\mathfrak{a} \in \mathfrak{A}, \mathfrak{b} \in \mathfrak{B}$ zu verstehen. Wir setzen voraus, daß der Nullpunkt und die Einheitspunkte in $\mathfrak{B}$ enthalten sind; dann ist offenbar $\mathfrak{Y} \leqq \mathfrak{C}$, und jeder natürliche Gitterpunkt $m$ läßt sich in der Form

$$
\mathfrak{m}=\mathfrak{b}_{1}+\cdots+\mathfrak{b}_{l(m)} \quad\left(\mathfrak{b}_{i} \in \mathfrak{B}\right)
$$

darstellen. Sei $l(m)$ die kleinste natürliche Zahl, für die eine solche Darstellung möglich ist. Es werde

$$
\overline{f i n}_{\mathfrak{n} \in \mathfrak{M}} \frac{1}{N(\mathfrak{n})} \sum_{\mathfrak{m} \in \mathfrak{M}(\mathfrak{n})} l(\mathfrak{m})=\lambda
$$

als mittlere Ordnung von $\mathfrak{B}$ bezeichnet. Ist $\lambda$ endlich, dann wird $\mathfrak{B}$ eine Basis endlicher Ordnung der natürlichen Gitterpunkte genannt. Dies sei im folgenden für $\mathfrak{B}$ der Fall.

9) Diese Definition der Dichte wird in 2-dimensionalen Fall bereits von L. CHEO [1] benutzt. 
Unter dieser Voraussetzung gilt

$$
\gamma \geqq \alpha\left(1+\frac{1}{2^{r}} \frac{1-2^{r-1} \alpha}{\lambda}\right) ;
$$

dies ist für $r=1$ die anfangs erwähnte Ungleichung von ERDös-LANDAU.

Wir können auf Grund dieser Ungleichung sagen, da $\beta \mathfrak{B}$ eine wesentliche Komponente für alle Mengen mit einer Dichte kleiner als $2^{-r+1}$ darstellt.

Zum Beweis wird analog zu den Überlegungen aus $\S 2$ geschlossen, wobei wir sogleich die Voraussetzung $\mathfrak{F}=\mathfrak{Q}=\mathfrak{A}$ machen. Sei $\mathfrak{n}$ ein fester natürlicher Gitterpunkt und $\mathfrak{m} \in \mathfrak{R}(\mathfrak{n})$. Dann sei

$$
\begin{aligned}
& D(\mathfrak{m}) \text { die Anzahl der verschiedenen Paare }(\mathfrak{a}, \overline{\mathfrak{a}}) \text { mit } \\
& \mathfrak{a} \in \mathfrak{A}(\mathfrak{l}), \overline{\mathfrak{a}} \in \overline{\mathfrak{A}}(\mathfrak{n}) \text { und } \overline{\mathfrak{a}}-\mathfrak{a}=\mathfrak{m} \text {; } \\
& E(\mathfrak{m}) \text { sei die Anzahl der verschiedenen Paare }\left(\mathfrak{a}^{\prime}, \mathfrak{a}\right) \text { mit } \\
& \mathfrak{a}^{\prime}, \mathfrak{a} \in \mathfrak{A}(\mathfrak{n}) \text { und } \mathfrak{a}^{\prime}-\mathfrak{a}=\mathfrak{m} .
\end{aligned}
$$

Damit gilt offenbar

$$
D(\mathfrak{m})+E(\mathfrak{m})=A(\mathfrak{n}-\mathfrak{m}) .
$$

Sei nun $t \in \mathfrak{R}(\mathfrak{m})$, dann besteht analog zum linearen Fall die Beziehung

$$
D(\mathrm{~m}) \leqq D(\mathrm{t})+D(\mathrm{~m}-\mathrm{t}) ;
$$

der Beweis erfolgt wie dort. Ebenfalls ist auch jetzt

$$
D(\mathfrak{b}) \leqq C(\mathfrak{n})-A(\mathfrak{n}) \quad(\mathfrak{b} \in \mathfrak{B}(\mathfrak{n})) .
$$

Wegen (5.2) erhält man folglich

Andererseits gilt

$$
\sum_{\mathfrak{m} \in \mathfrak{M}(\mathfrak{n})} D(\mathfrak{m}) \leqq \lambda N(\mathfrak{n})\{C(\mathfrak{n})-A(\mathfrak{n})\} .
$$

$$
\sum_{\mathfrak{m} \in \Re(\mathfrak{n})} E(\mathrm{~m}) \leqq\left(\begin{array}{c}
A(\mathfrak{n}) \\
2
\end{array}\right),
$$

wobei es sich jetzt im allgemeinen nicht wie im linearen Fall um eine Gleichung handeit.

Aus den bisherigen Angaben erhält man:

also

$$
\left\{\begin{array}{l}
\lambda \cdot N(\mathfrak{n})\{C(\mathfrak{n})-A(\mathfrak{n})\} \geqq \sum_{\mathfrak{m} \in \mathfrak{M}(\mathfrak{n})} A(\mathfrak{n}-\mathfrak{m})-\sum_{\mathfrak{m} \in \mathfrak{N}(\mathfrak{n})} E(\mathfrak{m}) \geq \\
\geq \sum_{\mathfrak{m} \in \mathfrak{M}(\mathfrak{n})} A(\mathfrak{n}-\mathfrak{m})-\left(\begin{array}{c}
A(\mathfrak{n}) \\
2
\end{array}\right) \geq \alpha \sum_{\mathfrak{m} \in \mathfrak{N}(\mathfrak{n})} N(\mathfrak{n}-\mathfrak{m})-\left(\begin{array}{c}
A(\mathfrak{n}) \\
2
\end{array}\right)
\end{array}\right.
$$

$$
\lambda \frac{C(\mathfrak{n})}{N(\mathfrak{l n})} \geq \lambda \frac{A(\mathfrak{n})}{N(\mathfrak{l n})}-\frac{A^{2}(\mathfrak{n})}{2 N^{2}(\mathfrak{l n})}+\frac{\alpha}{N^{2}(\mathfrak{n})}\left(\frac{N(\mathfrak{l})}{2}+\sum_{\mathfrak{m} \in \mathfrak{\Re}(\mathfrak{n})} N(\mathfrak{n}-\mathfrak{m})\right) .
$$


Da die Funktion $\xi\left(\lambda-\frac{\xi}{2}\right)$ mit $\alpha \leqq \xi \leqq 1$ und $\lambda \geqq 1$ ihren minimalen Wert für $\xi=\alpha$ annimmt, folgt aus dieser Ungleichung

$$
\lambda \frac{C(\mathfrak{n})}{N(\mathfrak{n})} \geqq \lambda \alpha-\frac{\alpha^{2}}{2}+\frac{\alpha}{N^{2}(\mathfrak{n})}\left(\frac{N(\mathfrak{n})}{2}+\sum_{\mathfrak{m} \in \mathfrak{M}(\mathfrak{n})} N(\mathfrak{n}-\mathfrak{m})\right) .
$$

Um (5.3) zu erhalten, haben wir jetzt $N(\mathfrak{n})$ und $\sum_{\mathfrak{m} \in \mathfrak{\Re}(\mathfrak{n})} N(\mathfrak{n}-\mathrm{m})$ zu berechnen. Offenbar ist

also gilt

$$
N(\mathfrak{n})=\prod_{i=1}^{r}\left(n_{i}+1\right)-1
$$

$$
\begin{aligned}
\sum_{\mathfrak{m} \in \mathfrak{M}(\mathfrak{n})} N(\mathfrak{n}-\mathfrak{m})=\sum_{\substack{\mathfrak{m} \in \mathfrak{M}(\mathfrak{n}) \\
\mathfrak{m} \neq \mathfrak{n}}} N(\mathfrak{m}) & =\sum_{m_{1}=0, \ldots, n_{1}} \ldots \sum_{m_{r}=0, \ldots, n_{r}}\left\{\prod_{i=1}^{r}\left(m_{i}+1\right)-1\right\}-N(\mathfrak{n})= \\
& =\prod_{i=1}^{r} \frac{\left(n_{i}+1\right)\left(n_{i}+2\right)}{2}-2 \prod_{i=1}^{r}\left(n_{i}+1\right)+1 .
\end{aligned}
$$

Daraus folgt

$$
\frac{N(\mathfrak{n})}{2}+\sum_{\mathfrak{m} \in \mathfrak{N}(\mathfrak{n})} N(\mathfrak{n}-\mathfrak{m})=\frac{1}{2^{r}} \prod_{i=1}^{r}\left(n_{i}+1\right)\left(n_{i}+2\right)-\frac{3}{2} \prod_{i=1}^{r}\left(n_{i}+1\right)+{ }_{2}^{1} .
$$

Zum Beweis von (5.3) ist jetzt wegen (5.7) nur noch nachzuweisen, daß dieser Ausdruck größer oder gleich $\frac{1}{2^{r}} N^{2}(\mathfrak{n})$ ist, und das ist offenbar der Fall, wenn die folgende Ungleichung gilt:

$$
\frac{1}{2^{r}} \prod_{i=1}^{r}\left(n_{i}+1\right)\left(n_{i}+2\right) \geq \frac{1}{2^{r}} \prod_{i=1}^{r}\left(n_{i}+1\right)^{2}+\left(\begin{array}{c}
3 \\
2
\end{array}-\frac{2}{2^{r}}\right) \prod_{i=1}^{r}\left(n_{i}+1\right) .
$$

Dabei kann $n_{i}>0(i=1, \ldots, r)$ vorausgesetzt werden, da für $n_{i}=0$ die $i$-tc Komponente keinen Beitrag liefert. Für $r=1$ ist (5.8) offenbar erfüllt:

$$
\frac{1}{2}(n+1)(n+2)=\frac{1}{2}(n+1)^{2}+\frac{1}{2}(n+1) .
$$

Der Beweis erfolgt nun durch Induktion nach $r$ mit dem Induktionsbeginn $r=2$. Für $r=2$ hat man wegen $n_{1} \geqq 1, n_{2} \geqq 1$ :

$$
\begin{aligned}
& { }_{4}^{1}\left(n_{1}+1\right)\left(n_{1}+2\right)\left(n_{2}+1\right)\left(n_{2}+2\right)> \\
& \quad>{ }_{4}^{1}\left(n_{1}+1\right)^{2}\left(n_{2}+1\right)^{2}+\left(\begin{array}{l}
3 \\
2
\end{array}-\frac{2}{4}\right)\left(n_{1}+1\right)\left(n_{2}+1\right) .
\end{aligned}
$$

Die Behauptung sei nun für $r$ richtig, dann folgt

$$
\begin{gathered}
\frac{1}{2^{r+1}} \prod_{i=1}^{r+1}\left(n_{i}+1\right)\left(n_{i}+2\right)=\frac{1}{2^{r}}\left(\prod_{i=1}^{r}\left(n_{i}+1\right)\left(n_{i}+2\right)\right) \frac{\left(n_{r+1}+1\right)\left(n_{r+1}+2\right)}{2} \geq \\
\geq \frac{1}{2^{r+1}} \prod_{i=1}^{r+1}\left(n_{i}+1\right)^{2}+\left(\frac{3}{2}-\frac{2}{2^{r}}\right) \frac{n_{r+1}+2}{2} \prod_{i=1}^{r+1}\left(n_{i}+1\right) .
\end{gathered}
$$




\section{Wegen}

$$
\left(\frac{3}{2}-\frac{2}{2^{r}}\right)^{n_{r+1}+2} \geq\left(\begin{array}{c}
3 \\
2
\end{array}-\frac{2}{2^{r}}\right)^{3} \frac{3}{2} \geq \frac{3}{2}-\frac{2}{2^{r+1}} \quad(r=2,3, \ldots)
$$

ist (5.8) auch für $r+1$ erfüllt. Damit ist (5.3) vollständig bewiesen.

\section{Literatur.}

[1] Cheo, L.: On the density of sets of Gaussian integers. Amer. Math. Monthly 58, 619-624 (1951). - [2] KASCH, F.: Abschätzung der Dichte von Summenmengen. Math. Z. 62, 36S-3S7 (1955). - [3] KNESER, M. : Abschätzung der asymptotischen Dichte von Summenmengen. Math. Z. 58, 459-484 (1953). - [4] Landau, E.: Über einige neuere Fortschritte der additiven Zahlentheorie. Cambridge Tracts No. 35. Cambridge 1937. - [5] RaIkov, D.: On the addition of point-sets in the sense of Schnirelmann (Russisch mit englischem Auszug). Mat. Sbornik $5(=47), 425-440$ (1939). — [6] SeL13ERG, S.: A survey of some recent results in additive number theory. Mat. Tidsskr. A 1949, 1-15.

Göttingen, Mathematisches Institut der Universität 